\title{
Paralisia cerebral Aspectos Fisioterapêuticos e Clínicos
}

\author{
Jaqueline Maria Resende Silveira Leite', Gilmar Fernandes do Prado²
}

\begin{abstract}
RESUMO
A paralisia cerebral congrega um grupo de afecções permanentes do sistema nervoso central sem caráter progressivo e de instalação no período neonatal. Há várias abordagens terapêuticas com possíveis benefícios ao paciente com paralisia cerebral, porém poucas embasadas em evidências científicas indiscutíveis.

A abordagem fisioterapêutica teria a finalidade de preparar a criança para uma função, manter ou aprimorar as já existentes, atuando sempre de forma a adequar a espasticidade. Entretanto, o prognóstico da paralisia cerebral depende evidentemente do grau de dificuldade motora, da intensidade de retrações e deformidades esqueléticas e da disponibilidade e qualidade da reabilitação.

Este artigo revisa aspectos clínicos da paralisia cerebral, discutindo a fisioterapia e as diversas abordagens terapêuticas utilizadas.
\end{abstract}

Descritores: Paralisia Cerebral, Espasticidade, Tratamento, Reabilitação.

\section{SUMMARY}

Cerebral palsy complies a group o permanent and no progressive central nervous system affection installed at neonatal and/or pre natal period. There are many ways to care and possible benefits to cerebral palsy patients, but we have almost no evidence-based approach to treat those patients.

Physical therapy could help the child preparing, keeping, or even improving functions, always targeting reduction of spasticity. Of course the cerebral palsy prognostic depends upon the degree of motor impairment, retractions, skeletal deformities, and availability and quality of rehabilitation service.

This paper intends to revise some clinical aspects of cerebral palsy, discussing the physical therapy and others therapeutics approaches performed.

Keywords: Cerebral Palsy, Spasticity, Treatment, Rehabilitation.

\section{Introdução}

Little, em 1843, descreveu, pela primeira vez, a encefalopatia crônica da infância, e a definiu como patologia ligada a diferentes causas e características, principalmente por rigidez muscular. Em 1862, estabeleceu a relação entre esse quadro e o parto anormal. Freud, em1897, sugeriu a expressão paralisia cerebral, que, mais tarde, foi consagrada por Phelps, ao se referir a um grupo de crianças que apresentavam transtornos motores mais ou menos severos devido à lesão do sistema nervoso central, semelhantes ou não aos transtornos motores da Síndrome de Little. ${ }^{19,20}$

Designa um grupo de afecções do SNC da infância que não têm caráter progressivo e que apresenta clinicamente distúrbios da motricidade, isto é, alterações do movimento, da postura, do equilíbrio, da coordenação com presença variável de movimentos involuntários.
A definição mais adotada pelos especialistas é de 1964 e caracteriza a PC como "um distúrbio permanente, embora não invariável, do movimento e da postura, devido a defeito ou lesão não progressiva do cérebro no começo da vida."

A paralisia cerebral (PC) é caracterizada por uma alteração dos movimentos controlados ou posturais dos pacientes, aparecendo cedo, sendo secundária a uma lesão, danificação ou disfunção do sistema nervoso central (SNC) e não é reconhecido como resultado de uma doença cerebral progressiva ou degenerativa. ${ }^{1,2} \mathrm{O}$ evento lesivo pode ocorrer no período pré, peri ou pósnatal. ${ }^{1}$

As encefalopatias crônicas da infância incluem numerosas afecções com várias etiologias e quadros clínicos diversos tendo em comum o fato de afetarem o sistema nervoso central das crianças com um caráter 
crônico.Um subgrupo de pacientes é formado por afecções de caráter progressivo que vão se agravando lenta ou rapidamente, e outro subgrupo apresenta caráter não progressivo e tendência à regressão espontânea, maior ou menor, à medida que o tempo vai passando.

Embora o desenvolvimento da criança normal seja a base sobre a qual o desenvolvimento anormal é avaliado, disto não decorre que a avaliação e o tratamento devam depender de uma aderência estrita aos estágios de desenvolvimento normal. Mesmo crianças normais mostram muitas variações das seqüelas de desenvolvimento normal e dos padrões de desenvolvimento estabelecidos para a criança média. A criança com paralisia cerebral mostrará variações adicionais em virtude das dificuldades neurológicas e mecânicas.

\section{Incidência}

A incidência das moderadas e severas está entre 1,5 e 2,5 por 1000 nascidos vivos nos países desenvolvidos; mas há relatos de incidência geral, incluindo todas as formas de 7:1000. ${ }^{1}$ Nestes países, calcula-se que em relação à crianças em idade escolar freqüentando centros de reabilitação, a prevalência seja de $2 / 1000$. Na Inglaterra admite-se a existência de 1,5/1000 pacientes. No Brasil não há estudos conclusivos a respeito e a incidência depende do critério diagnóstico de cada estudo, sendo assim, presume-se uma incidência elevada devido aos poucos cuidados com as gestantes.

Nos EUA, admite-se a existência de 550 a 600 mil pacientes sendo que há um aumento de 20 mil novos casos a cada ano.

Segundo Salter (1985), há muitas causas de paralisia cerebral; e qualquer condição que leve a uma anormalidade do cérebro pode ser responsável. As causas mais comuns são: desenvolvimento congênito anormal do cérebro, particularmente do cerebelo; anóxia cerebral perinatal, especialmente quando associada com prematuridade; lesão traumática do cérebro, no nascimento, geralmente decorrente de trabalho de parto prolongado, ou uso de fórceps; eritroblastose por incompatibilidade Rh; infecções cerebrais (encefalite) na fase inicial do período pós-natal.

Entretanto, como a paralisia cerebral é raramente diagnosticada até pelo menos vários meses após o nascimento, a causa precisa da lesão cerebral numa criança é freqüentemente especulativa.

\section{Tipos de PC}

As crianças com PC têm como principal característica o comprometimento motor, que influencia no seu desempenho funcional. De acordo com Schwartzman (1993) e Souza \& Ferraretto (1998) ${ }^{1}$, a PC pode ser classificada por dois critérios: pelo tipo de disfunção motora presente, ou seja, o quadro clínico resultante, que inclui os tipos extrapiramidal ou discinético (atetóide, coréico e distônico), atáxico, misto e espástico; e pela topografia dos prejuízos, ou seja, localização do corpo afetado, que inclui tetraplegia ou quadriplegia, monoplegia, paraplegia ou diplegia e hemiplegia. Na PC, a forma espástica é a mais encontrada e freqüente em $88 \%$ dos casos. ${ }^{3,4}$

\section{Diagnóstico}

O diagnóstico de PC usualmente envolve retardo ou atraso no desenvolvimento motor, persistência de reflexos primitivos, presença de reflexos anormais, e o fracasso do desenvolvimento dos reflexos protetores, tal como a resposta de pára-quedas, caracterizada pela extensão dos braços como se a criança fosse apoiar-se e com isso apoio do corpo sobre os braços. ${ }^{2}$

Uma anamnese e exame físico minuciosos devem eliminar a possibilidade de distúrbios progressivo do sistema Nervoso Central, incluindo as doenças degenerativas, tumor da medula espinhal ou distrofia muscular. De acordo com a intensidade e a natureza das anormalidades neurológicas, um eletroencefalograma (EEG) e tomografia computadorizada (TC) iniciais podem estar indicados para determinar a localização e extensão das lesões estruturais ou malformações congênitas associadas. Exames adicionais podem incluir testes das funções auditiva e visual. Como a Paralisia Cerebral geralmente está associada a um amplo espectro de distúrbios do desenvolvimento, uma abordagem multidisciplinar é mais benéfica na avaliação e tratamento dessas crianças.

Os médicos que atendem a criança com PC dispõem de uma série de procedimentos de intervenção com objetivos específicos a serem atingidos. Os procedimentos utilizados para melhora da espasticidade das crianças com PC são a eliminação de fatores agravantes da espasticidade, as terapias de reabilitação, as órteses, a farmacoterapia oral, os desnervadores químicos, as cirurgias ortopédicas e a neurocirurgia.

\section{Quadro Clínico}

Na observação clínica da paralisia cerebral, devese levar em consideração a extensão do distúrbio motor, sua intensidade e, principalmente, a caracterização semiológica desse distúrbio. Assim a paralisia cerebral apresenta várias formas clínicas.

1.Hemiplegia : É a manifestação mais freqüente, com maior comprometimento do membro superior; acompanha-se de sinais de liberação tais como espasticidade, hiper-reflexia e sinal de Babinski. O paciente assume atitude em semiflexão do membro superior, permanecendo o membro inferior hiperestendido e aduzido, e o pé em postura eqüinovara. É comum hipotrofia dos segmentos acometidos, sendo também possível a ocorrência de outras hemi-hipoestesia ou hemianopsia.

2. Hemiplegia bilateral (tetra ou quadriplegia) : Ocorrem de 9 a $43 \%$ dos pacientes. Ocorrem lesões difusas bilateral no sistema piramidal dando além da grave tetraparesia espástica com intensas retrações em semiflexão, síndrome pseudobulbar (hipomimia, disfagia e disartria), podendo ocorrer ainda microcefalia, deficiência mental e epilepsia.

3. Diplegia : Ocorre em 10 a $30 \%$ dos pacientes, sendo a forma mais encontrada em prematuros. Trata-se de um comprometimento dos membros inferiores, comumente evidenciando uma acentuada hipertonia dos adutores, que configura em alguns doentes o aspecto semiológico denominado síndrome de Little (postura com cruzamento dos membros inferiores e marcha "em tesoura"). Há 
diferentes gradações quanto à intensidade do distúrbio, podendo ser pouco afetado (tendo recuperação e bom prognóstico - adaptam-se à vida diária); enquanto outros evoluem mal com graves limitações funcionais. Os dados semiológicos são muito variáveis. No $1^{\circ}$ ano de vida, a criança apresenta-se hipotônica, evoluindo gradativamente para uma outra fase em que se observa um quadro de distonia intermitente, com tendência ao opistótono quando estimulada. Nos casos mais graves a criança pode permanecer num destes estágios por toda a sua vida, porém geralmente passa a exibir hipertonia espástica, inicialmente extensora e, finalmente, com graves retrações semiflexoras.

4. Discinesia : Atualmente é a mais rara, pois manifesta-se através de movimentos involuntários, sobretudo distonias axiais e/ou movimentos córeoatetóides das extremidades. No primeiro ano de vida este padrão costuma não estar definido, podendo existir hipotonia muscular. Em geral, quando estes pacientes estão relaxados a movimentação passiva é facilitada.

5. Ataxia : Igualmente rara. Inicialmente pode traduzirse por hipotonia e, aos poucos, verificam-se alterações do equilíbrio (ataxia axial) e, menos comumente, da coordenação ( ataxia apendicular). Sua marcha se faz com aumento da base de sustentação podendo apresentar tremor intencional.

6. Formas mistas : É a associação das manifestações anteriores, correspondendo, geralmente, ao encontro de movimentos distônicos e córeo-atetóides ou à combinação de ataxia com plegia (sobretudo diplegia). No total, cerca de $75 \%$ dos pacientes doentes com paralisia cerebral apresentam padrão espástico.

Além do distúrbio motor, obrigatório para a caracterização da paralisia cerebral, o quadro clínico pode incluir também outras manifestações acessórias com freqüência variável: 1.Deficiência mental: Ocorre de 30 a $70 \%$ dos pacientes. Está mais associada às formas tetraplégicas, diplégicas ou mistas; 2. Epilepsia: Varia de 25 a $35 \%$ dos casos, ocorrendo mais associado com a forma hemiplégica ou tetraplégica; 3. Distúrbios da linguagem; 4. Distúrbios visuais : Pode ocorrer perda da acuidade visual ou dos movimentos oculares (estrabismo); 5. Distúrbios do comportamento : São mais comuns nas crianças com inteligência normal ou limítrofe, que se sentem frustradas pela sua limitação motora, quadro agravado em alguns casos pela super proteção ou rejeição familiar; 6. Distúrbios ortopédicos : Mesmo nos pacientes submetidos à reabilitação bem orientada, são comuns retrações fibrotendíneas $(50 \%)$ cifoescoliose (15\%), "coxa valga"(5\%) e deformidades nos pés.

Todos esses distúrbios se dão devido a alterações nas áreas motoras cerebrais específicas durante a infância.

\section{Tratamento}

O tratamento é paliativo, visto que não se pode agir sobre uma lesão já superada e cicatricial.

O Tratamento medicamentoso limita-se, em geral, ao uso de anticonvulsivantes, quando necessários e mais raramente medicamentos psiquiátricos para tentar o controle dos distúrbios afetivos-emocionais e da agitação psicomotora ligada à deficiência mental. medicamentos mais utilizados no tratamento da espasticidade são o baclofen, o diazepam, o clonazepan, dantrolene, a clonidina, a tizanidina, a clopromazina e também a morfina. ${ }^{3,15}$ No Centro Cochrane encontra-se uma revisão sistemática de medicamento antiespásticos, que compara a ação de drogas mais usadas como, diazepam, dantrolene e baclofen com outras dogas. ${ }^{20}$

O baclofen é agonista do GABA, atuando em nível de receptores $G A B A-B$, inibindo o influxo pré-sináptico de cálcio, que bloqueará a liberação de neurotransmissores excitatórios, na medula espinhal. O baclofen é mais eficaz para reduzir a espasticidade causada por lesões da medula espinhal do que de lesões cerebrais. São utilizadas doses progressivas, de até $80-120 \mathrm{mg}$ ao dia, podendo chegar a160 mg ao dia.. Os efeitos colaterais são sonolência, tontura, mal-estar, ataxia, confusão mental, cefaléia, depressão respiratória e cardiovascular e alucinações. $3,15,23$

Os benzodiazepínicos atuam nos receptores GABA pré e pós-sinápticos (aumentando a afinidade dos receptores GABA por GABA endógeno), aumentando a inibição sináptica ao nível espinhal e supra-espinhal. Utilizam-se o diazepam e clonazepam. O diazepam em doses de até $60 \mathrm{mg}$ ao dia, enquanto o clonazepam em doses de até $18 \mathrm{mg}$ ao dia. Os efeitos colaterais são muito semelhantes aos do baclofen, principalmente a sedação, sonolência, astenia e agitação paradoxal. ${ }^{3,15,23}$

O dantrolene sódico é medicamento de ação periférica, em nível muscular, através do bloqueio da liberação de cálcio no retículo sarcoplasmático, com subseqüente inibição da despolarização. As doses preconizadas estão entre 50 a $800 \mathrm{mg}$ ao dia. Os efeitos colaterais são tontura, sonolência, letargia e diarréia. ${ }^{3,15}$

A clonidina é agonista de receptores alfa-2 adrenérgicos, localizados principalmente na substância gelatinosa e nas colunas de células intermédio-laterais da medula espinhal torácica. É uma medicação coadjuvante para o tratamento da espasticidade, principalmente a de origem espinhal. As doses podem chegar até $0,4 \mathrm{mg}$ ao dia. O efeito colateral mais comum é a hipotensão postural. . $^{3,15}$

A tizanidina é um agonista alfa-2 adrenérgico que provoca depressão indireta nos reflexos polissináptico, por facilitar a ação da glicina,um neurotransmissor inibitório, e por reduzir pré-sinapticamente a liberação de neurotransmissores excitatórios. As doses devem ser aumentadas de $2 \mathrm{mg}$ ao dia até $36 \mathrm{mg}$ ao dia. Apresenta menos efeitos colaterais do que o baclofen e o diazepam, os efeitos colaterais são: sedação, boca seca, sonolência, astenia, tontura, alucinações visuais e hipotensão. 3,15,22

Os fenotiazínicos, tais como a clorpromazina, têm demonstrado eficácia no tratamento da espasticidade, atuando como bloqueador dos receptores alfaadrenérgicos. Os efeitos colaterais comuns como a sedação e a possibilidade de discinesia tardia, contraindicam a sua utilização. . $^{3,15}$

A morfina pode ser utilizada por via intratecal para o tratamento da espasticidade. Promove, assim, a inibição dos reflexos polissinápticos da medula espinhal, através da ação em receptores opióides. A possibilidade de provocar o desenvolvimento de tolerância e dependência reduz a sua utilização. ${ }^{3,15}$ 
Os bloqueios neuromusculares com álcool, fenol e anestésicos locais têm sido utilizados há anos. Atualmente, a toxina botulínica, em casos selecionados, tem demonstrado utilidade na prevenção de deformidades secundárias à espasticidade, modificando a qualidade de ida das crianças com paralisia cerebral. ${ }^{19}$

O Tratamento cirúrgico envolve cirurgias ortopédicas de deformidades e para estabilização articular, que devem ao mesmo tempo preservar a função e aliviar a dor.

A Reabilitação admite múltiplas possibilidades dependendo da análise cuidadosa de cada paciente individualmente. O principal papel do neuropediatra é estabelecer quais as prioridades de tratamento para cada criança em cada época do desenvolvimento. Deve-se também reconhecer aqueles casos graves nos quais é inútil insistir com terapias dispendiosas que geram ansiedade e expectativa na família, sem alterar o prognóstico da criança, a qual seria melhor mantida apenas com fisioterapia ou em instituição.

A fisioterapia tem como objetivo a inibição da atividade reflexa anormal para normalizar o tônus muscular e facilitar o movimento normal, com isso haverá uma melhora da força, da flexibilidade, da amplitude de movimento (ADM), dos padrões de movimento e, em geral, das capacidades motoras básicas para a mobilidade funcional. As metas de um programa de reabilitação são reduzir a incapacidade e otimizar a função. Atualmente não há evidências suficientes que indiquem que as técnicas de facilitação e inibição, ou as técnicas de facilitação neuromuscular proprioceptivas são superiores umas às outras, e os exercícios tradicionais menos custosos ${ }^{5,3}$. Sendo assim, os alongamentos músculotendinosos devem ser lentos e realizados diariamente para manter a amplitude de movimento e reduzir o tônus muscular 16, 17. Exercícios frente a grande resistência podem ser úteis para fortalecer músculos débeis, mas devem ser evitados nos casos de pacientes com lesões centrais, pois nestes se reforçarão as reações tônicas anormais já existentes e conseqüentemente aumentará a espasticidade ${ }^{15}$.

Em 1990, a seção Pediátrica da Associação Americana de Terapia Física emitiu uma declaração onde concluía que não havia evidência alguma de que a terapia física diminuiria o tônus muscular e os reflexos primitivos ${ }^{6,3}$.

Segundo Girolami e Campbell ${ }^{3}$ em um estudo controlado e randomizado, comparou-se o método neuroevolutivo Bobath e a manipulação inespecífica de crianças prematuras, e observaram que o tratamento neuroevolutivo melhorou o controle postural,o que não foi visto sobre o tônus muscular e os reflexos primitivos.

Existem quatro categorias de intervenção, as quais devem apresentar uma combinação para suprir todos os aspectos das disfunções dos movimentos nas crianças com Paralisia Cerebral ${ }^{18}$ : a) enfoque biomecânico; b) enfoque neurofisiológico; c) enfoque do desenvolvimento; e d) enfoque sensorial.

O enfoque biomecânico aplica os princípios da cinética e da cinemática para os movimentos do corpo humano. Incluem movimento, resistência e as forças necessárias para melhorar as atividades de vida diária.

O enfoque neurofisiológico e do desenvolvimento são realizados juntos, recebendo o nome de enfoque neuroevolutivo. Este enfoque inclue uma combinação de técnicas neurofisiológicas e do conhecimento da seqüência do desenvolvimento, como se observa no tratamento de Rood, de Brunnstrom, na facilitação neuromuscular proprioceptiva (Kabat) e no tratamento neuroevolutivo Bobath ${ }^{18}$.

As técnicas de tratamento sensorial promovem experiências sensoriais apropriadas e variadas (tátil, proprioceptiva, cinestésica, visual, auditiva, gustativa, etc.) para as crianças com espasticidade facilitando assim uma aferência motora apropriada.

\section{Prognóstico}

O prognóstico depende evidentemente do grau de dificuldade motora, da intensidade de retrações e deformidades esqueléticas e da disponibilidade e qualidade da reabilitação. Entretanto, mesmo que o quadro motor seja considerado de bom prognóstico, existem três outros fatores que interferem decisivamente no futuro desempenho da criança: o grau de deficiência mental, o número de crises epilépticas e a intensidade do distúrbio de comportamento. É evidente que as crianças com deficiência mental moderada ou grave, com epilepsia de difícil controle ou com atitudes negativistas ou agressivas, não tem condições de responder a reabilitação.

\section{Conclusão}

A criança portadora de Paralisia Cerebral exibe os resultados complexos de uma lesão do cérebro ou de um erro do desenvolvimento cerebral. À medida que a criança cresce e evolui, outros fatores se combinam com os efeitos da lesão para agravar as deficiências funcionais. Esses fatores fazem parte dos efeitos da falta de atividade sobre a flexibilidade do sistema osteomuscular assim como os efeitos que uma série de atividade muscular limitada e estereotipada exercem sobre o sistema nervoso.

Ainda não se tem um fator determinante para a etiologia na Paralisia Cerebral. As condições que mais contribuem são a hipoxemia e a isquemia, dependendo de sua intensidade e do período gestacional.

O fisioterapeuta que atende crianças com Paralisia Cerebral tem de se responsabilizar de estar sempre familiarizado com os atuais avanços da intervenção em sua prática diária, para que somente utilize aqueles procedimentos cuja eficácia responda a evidências científicas.

Até o momento não existem evidências dos benefícios da reabilitação física para o tratamento da espasticidade. Em alguns casos, um programa de reabilitação física deverá atentar a outros fatores que não somente ao desenvolvimento motor, como também oferecer estratégias mais práticas e fáceis.

A fisioterapia na criança deve consistir no treinamento específico de atos como: levantar-se, dar passos ou caminhar, sentar-se, pegar e manusear objetos, além de exercícios destinados a aumentar a força muscular e melhorar o controle sobre os movimentos. Em resumo, a fisioterapia prepara a criança para uma função, mantém as já existentes ou as aprimora, trabalhando sempre com a finalidade de reduzir a espasticidade. 


\section{REFERÊNCIAS BIBLIOGRÁFICAS}

1. Ferraretto, Ivan \& Souza, Ângela M. C. Paralisia Cerebral - aspectos práticos. São Paulo: Memnon, 1998

2. Russman et al. Cerebral Palsy: A Rational Approach to a Treatment Protocol, and the Role of Botulinum Toxin in Treatment. Muscle \& Nerve, Suppl.6, 1997.

3. González, R. C. \& Sepúlveda, R. F. C. Tratamiento de La Espasticidad en Parálisis Cerebral con Toxina Botulínica. Rev. Neurol, 34 (1), 2002.

4. Young, R. R. Spasticity: a review. Neurology, 44 (Suppl 9), 1994.

5. Williams, P. E. \& Goldspick, G. The effect of immobilization on the longitudinal growth of striated muscle fibers. J. Anatomy, 116, 1973.

6. O'dwyer, N. J. et al. Mechanisms of muscle growth related to muscle contracture in cerebral palsy. Dev Med Child Neurol, 31, 1989.

7. Nitrini, Ricardo \& Bacheshi, Luiz A. A neurologia que todo médico deve saber, 4ª ed., São Paulo: Santos, 1999.

8. Behrmar, R.E. et al. Tratado de Pediatria; 15a edição, Rio de Janeiro, Ed. Guanabara Koogan, 1997 (Vol.II).

9. Marcondes, E.; Pediatria básica; $8^{a}$ edição, São Paulo, Savier, 1991, vol.2.

10. Ratliffe, Katherine T. Fisioterapia na clínica pediátrica. $1^{a}$ ed. São Paulo: Santos, 2000.

11. Salter, Robert. Distúrbios e lesões do sistema músculo-esquelético.2a ed. São Paulo: Medsi,,,1985.

12. Shepherd, Roberta B. Fisioterapia em pediatria. $3^{\text {a }}$ ed. São Paulo: Santos, 1995.

13. Cambier, J. ; MASSON, M.; DEHEN, H. Manual de Neurologia, $2^{a}$ ed. São Paulo, Atheneu. 1988.

14. Flehmig, Inge. Texto e Atlas do Desenvolvimento Normal e seus Desvios no Lactente: diagnóstico e tratamento precoce do Nascimento até o $18^{\circ}$ mês. São Paulo: Atheneu. 2000.

15. Teive, H. A. G. et al. Tratamento da espasticidade: uma atualização. Arq Neuropsiquiat 1998; 56 (4): 852 - 858.

16. Bobath, B. Hemiplegia no Adulto

17. Stokes, M. Neurologia para Fisioterapeutas. São Paulo: Premier, 2000.

18. Helsel, P, McGee, J, Graveline CH. Physical Management of Spasticity. J Child Neurol 2001; 16: $24-30$.

19. Rotta, N. T. Paralisia cerebral, novas perspectivas terapêuticas. J Pediatr (Rio J) 2002; 78 (Supl.1): S48 - S54.

20. Diament, A \& Cypel, A. Neurologia Infantil. $3^{a}$ ed. São Paulo, Atheneu.1996. p.781-98.

21. Howad,D.C. Anti Spastic Medication for Spasticity in Cerebral Palsy (Protocol). The Cochrane Library, Issue 4, 2002.

22. Davidoff, R.A. Antispasticity Drugs: Mechanisms of Action. Ann Neurol, 17: 107-116, 1985.

23. Milla, P.J. \& Jackson, A.D.M. A Controlled Trial of Baclofen in Children with Cerebral Palsy. J Int Med Res, 5: 398404, 1977. 